\title{
Antioxidant Activity of Hydroxystilbene Derivatives in Homogeneous Solution
}

Riccardo Amorati, Marco Lucarini, Veronica Mugnaini, Gian Franco Pedulli

Dipartimento di Chimica Organica “A. Mangini”"

Via S. Donato 15, 40127 Bologna, Italy

Marinella Roberti, Daniela Pizzirani

Dipartimento di Scienze Farmaceutiche

Via Belmeloro 6, 40126 Bologna, Italy

\section{Table of Contents for the Supporting Information}

${ }^{1} \mathrm{HNMR}$ of compound $\mathbf{5 c}$ pages S2, S3.

${ }^{13} \mathrm{CNMR}$ of compound $\mathbf{5 c}$ pages $\mathrm{S} 4, \mathrm{~S} 5$.

${ }^{1} \mathrm{HNMR}$ of compound $\mathbf{5 t}$ pages $\mathrm{S} 6, \mathrm{~S} 7$.

${ }^{13} \mathrm{CNMR}$ of compound $\mathbf{5 t}$ page $\mathrm{S} 8$.

${ }^{1}$ HNMR of compound $6 \mathbf{t}$ pages S9, S10.

${ }^{13} \mathrm{CNMR}$ of compound $\mathbf{6 t}$ page $\mathrm{S} 11$.

Table S1 pages S12, S13.

Figure S1 page S13.

Geometries and Total Energies pages S14-S31. 


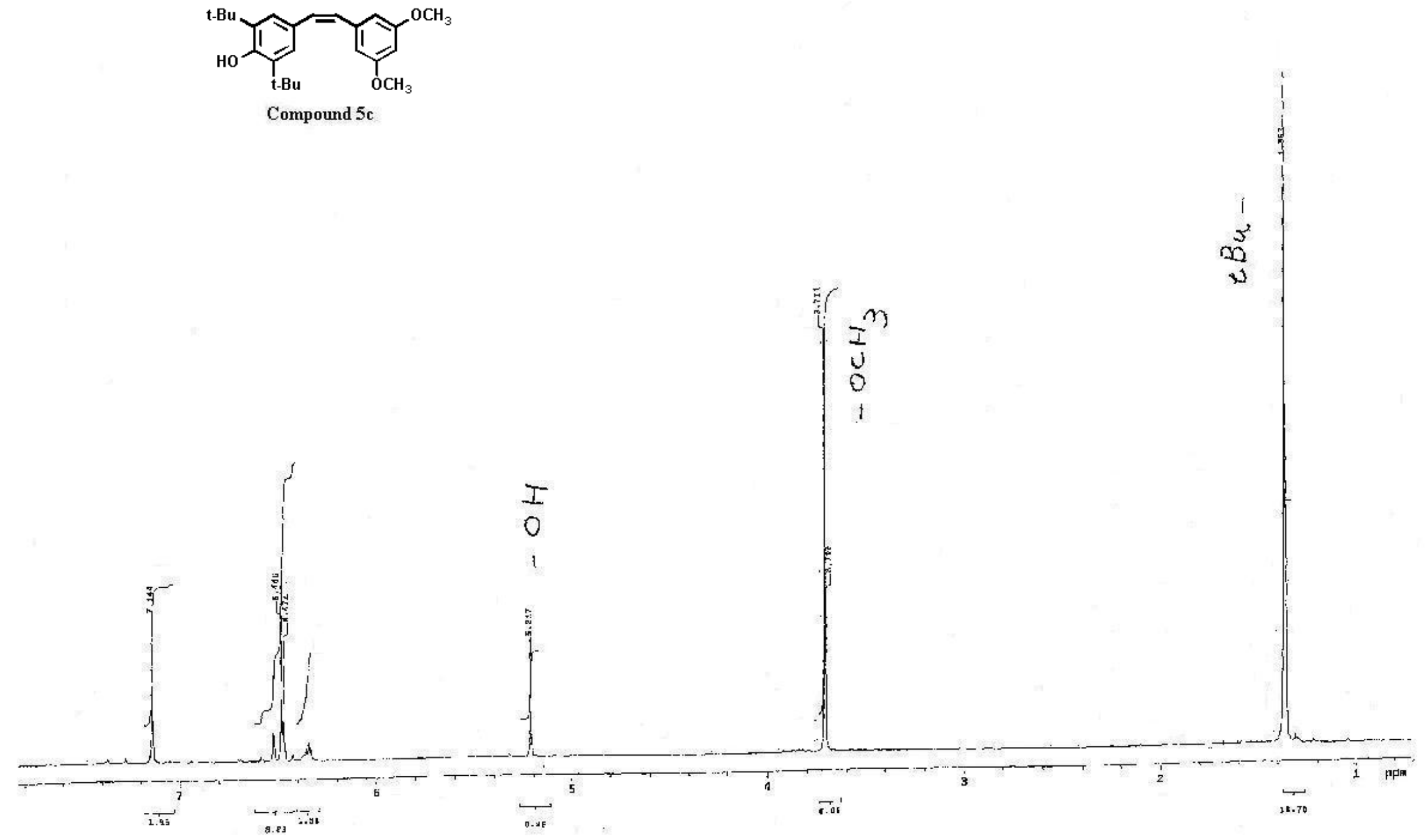



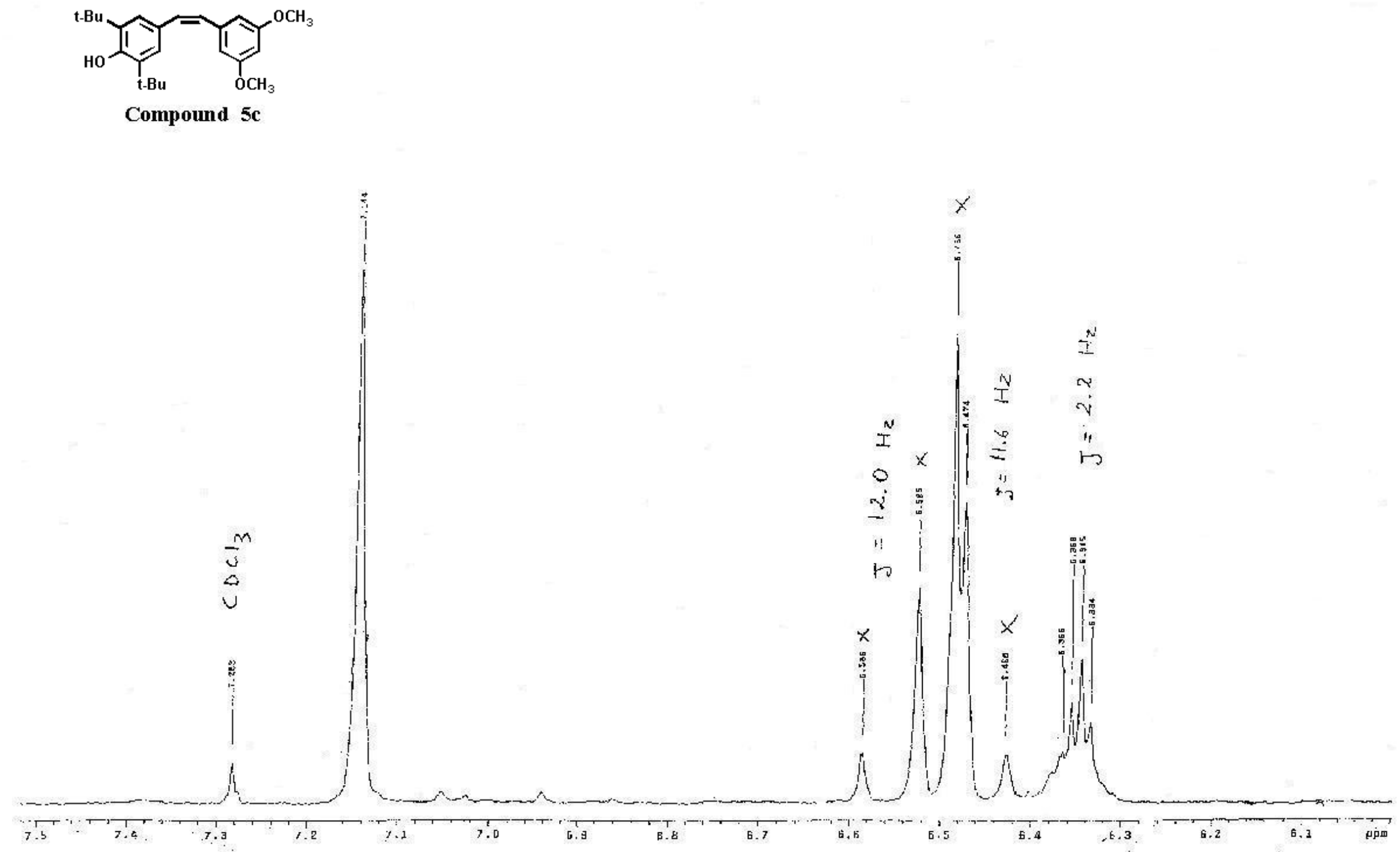


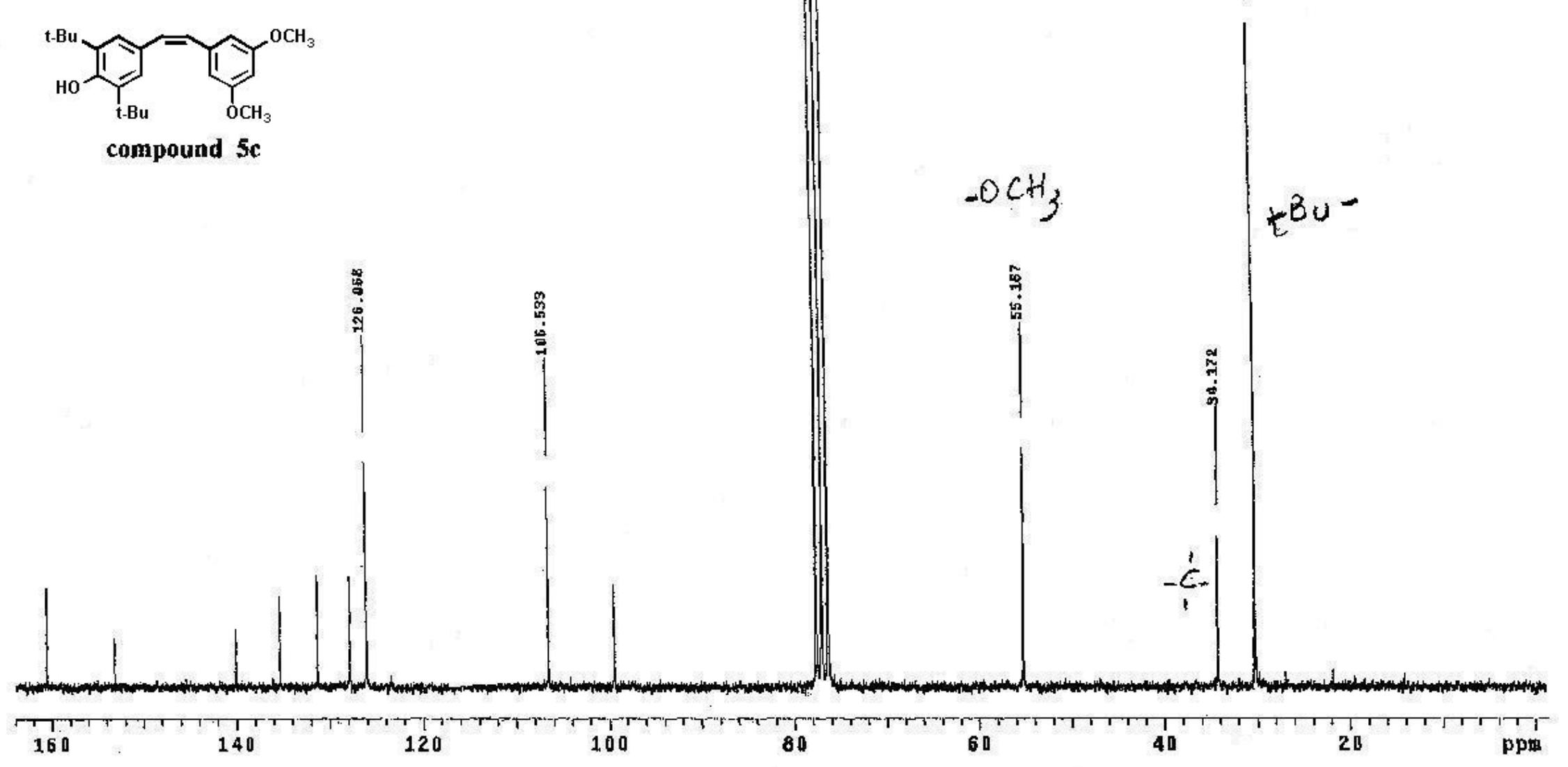

S4 


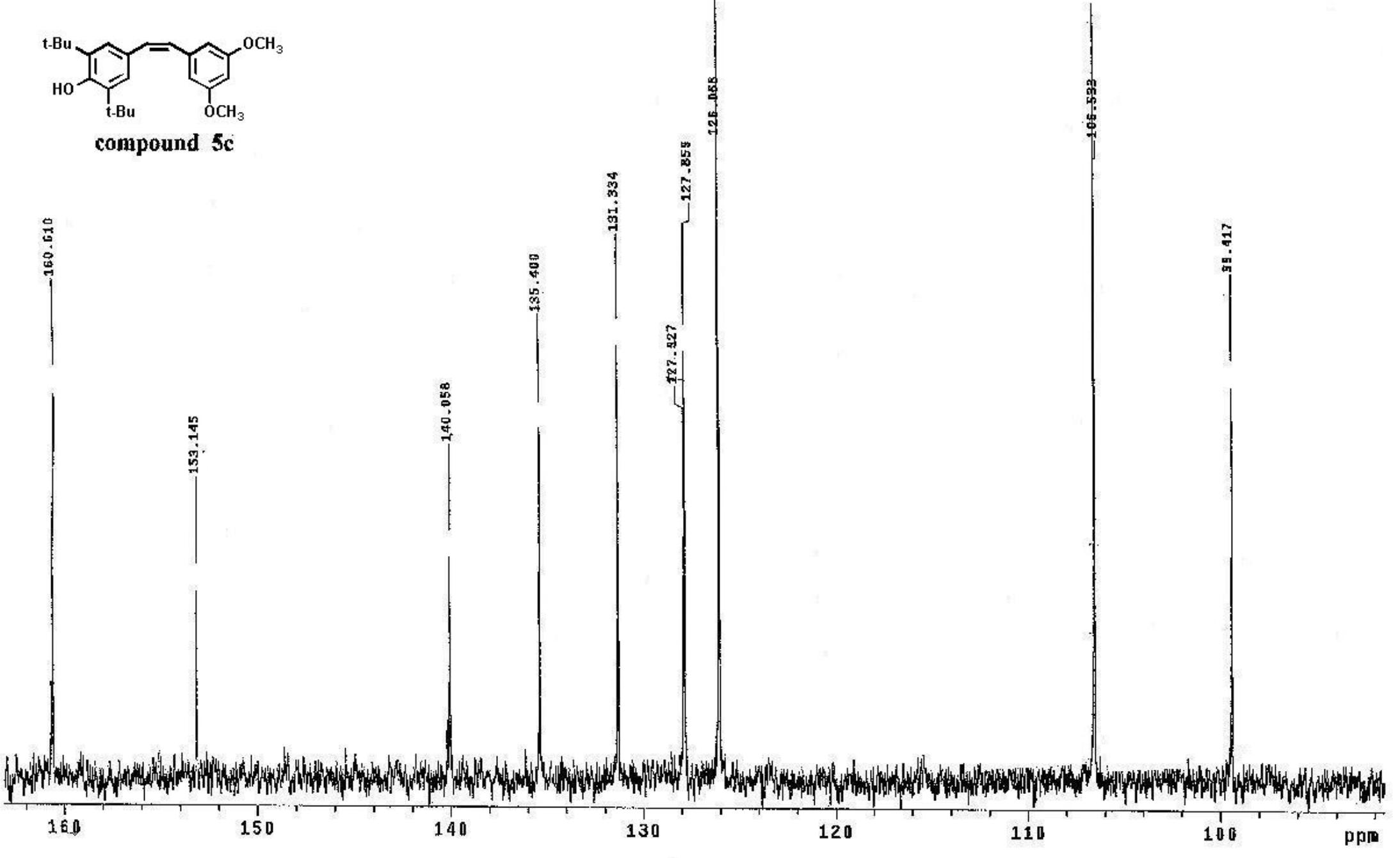

S5 


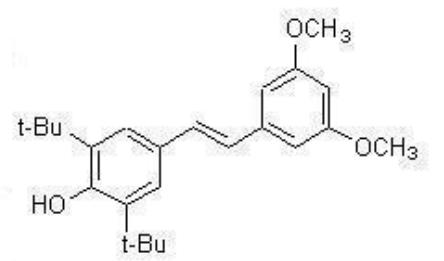

Compound 5t

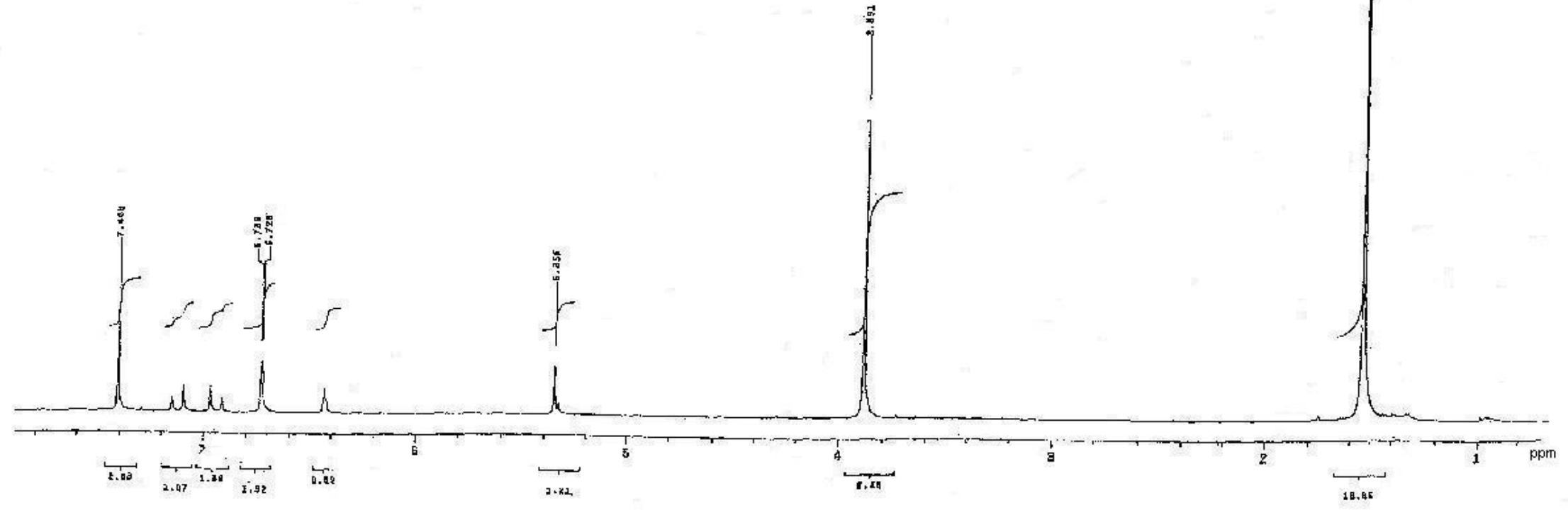




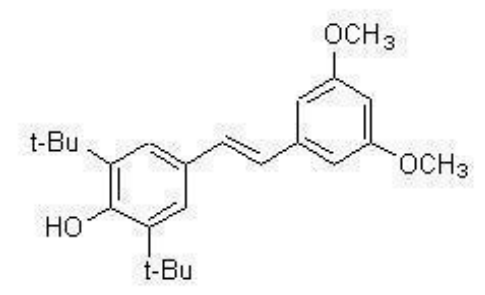

Compound $5 t$

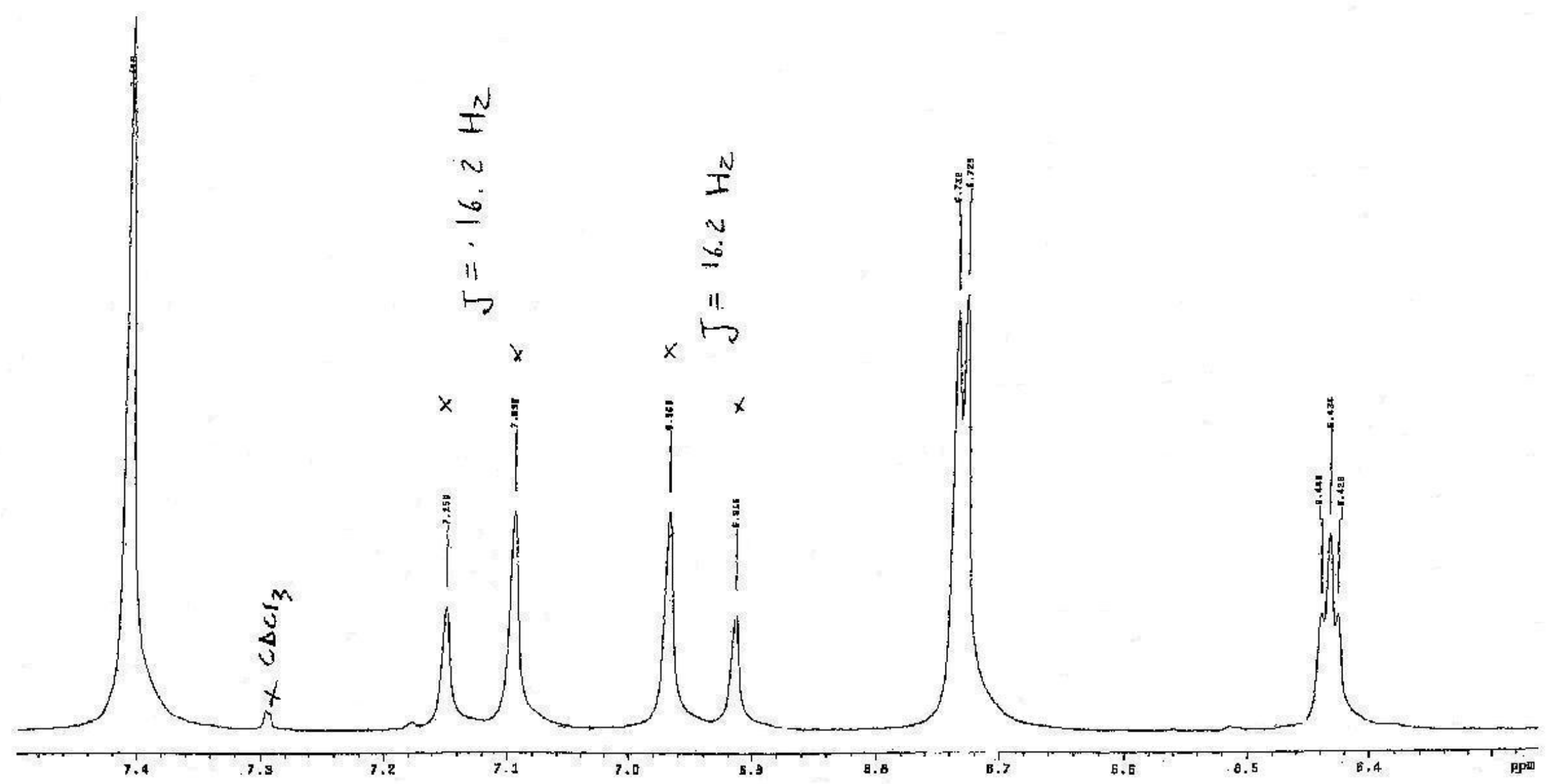



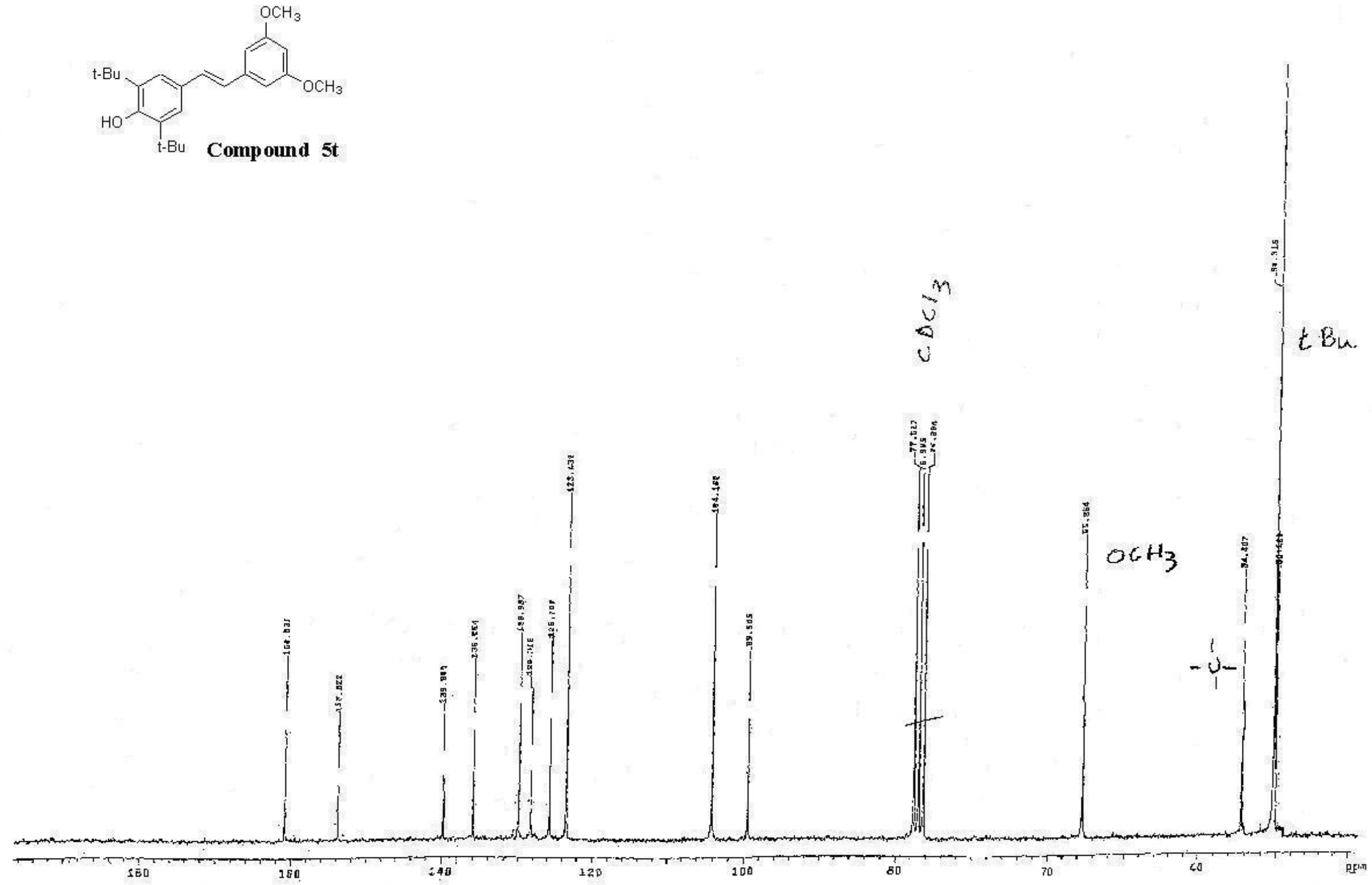


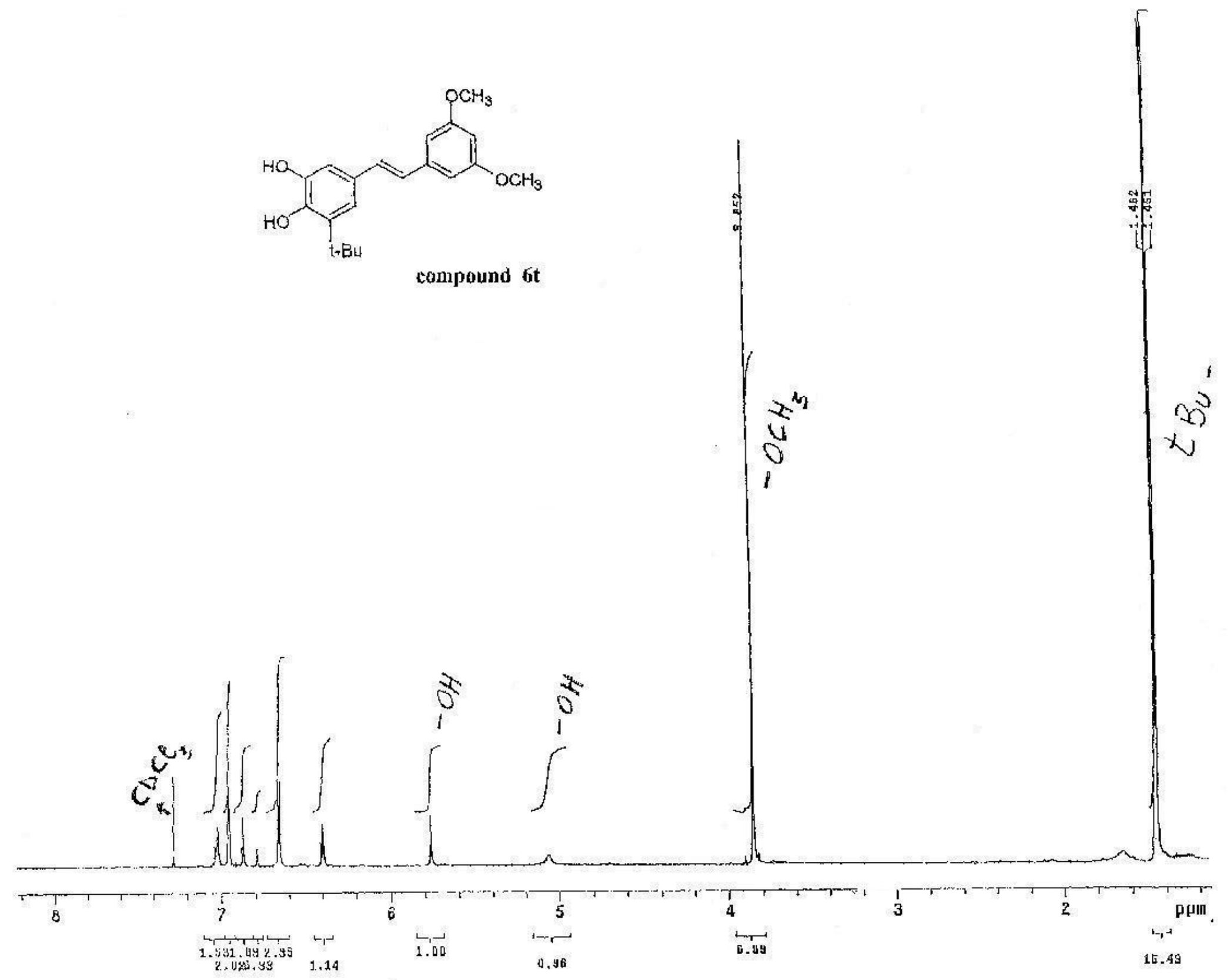




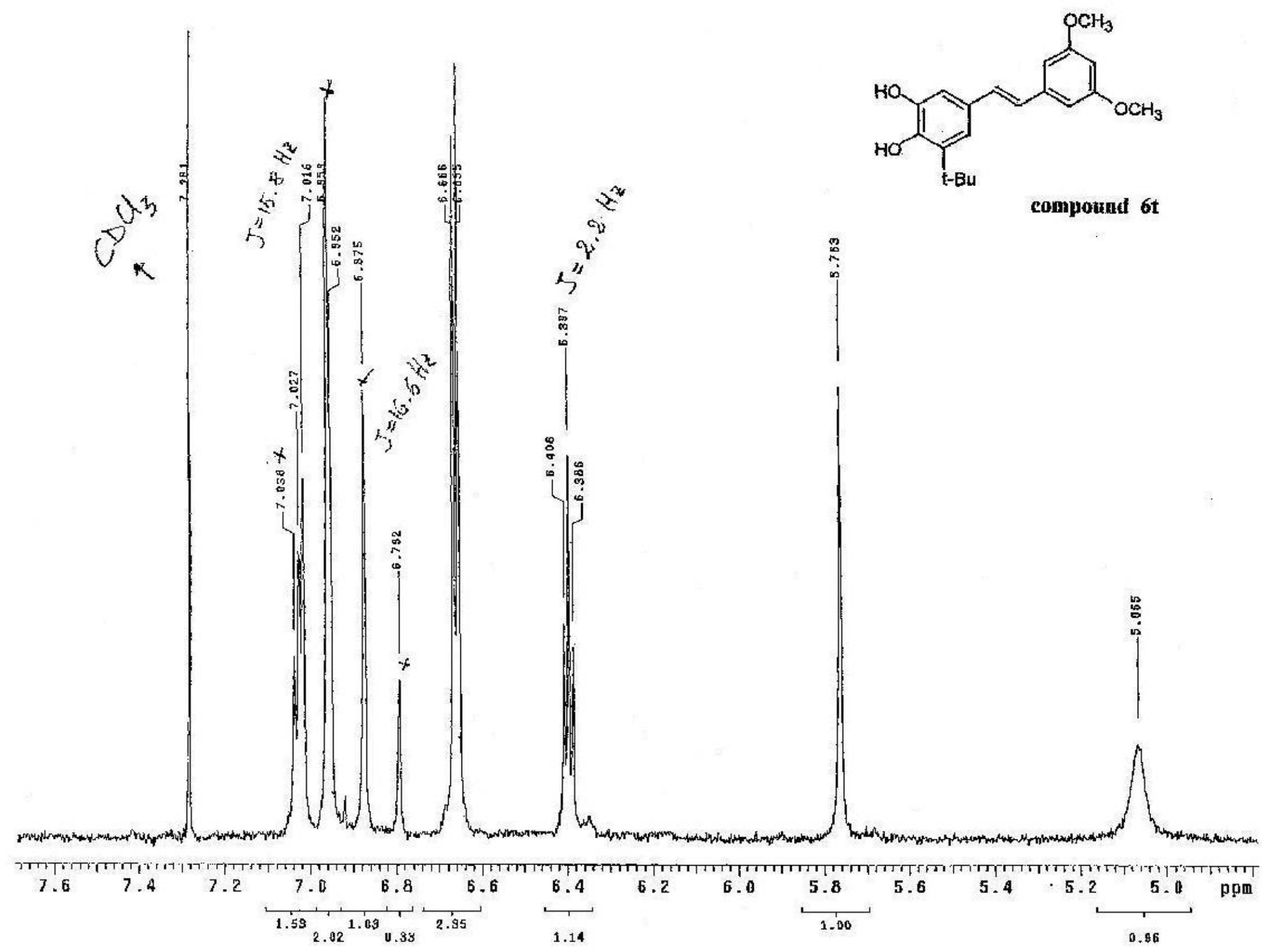




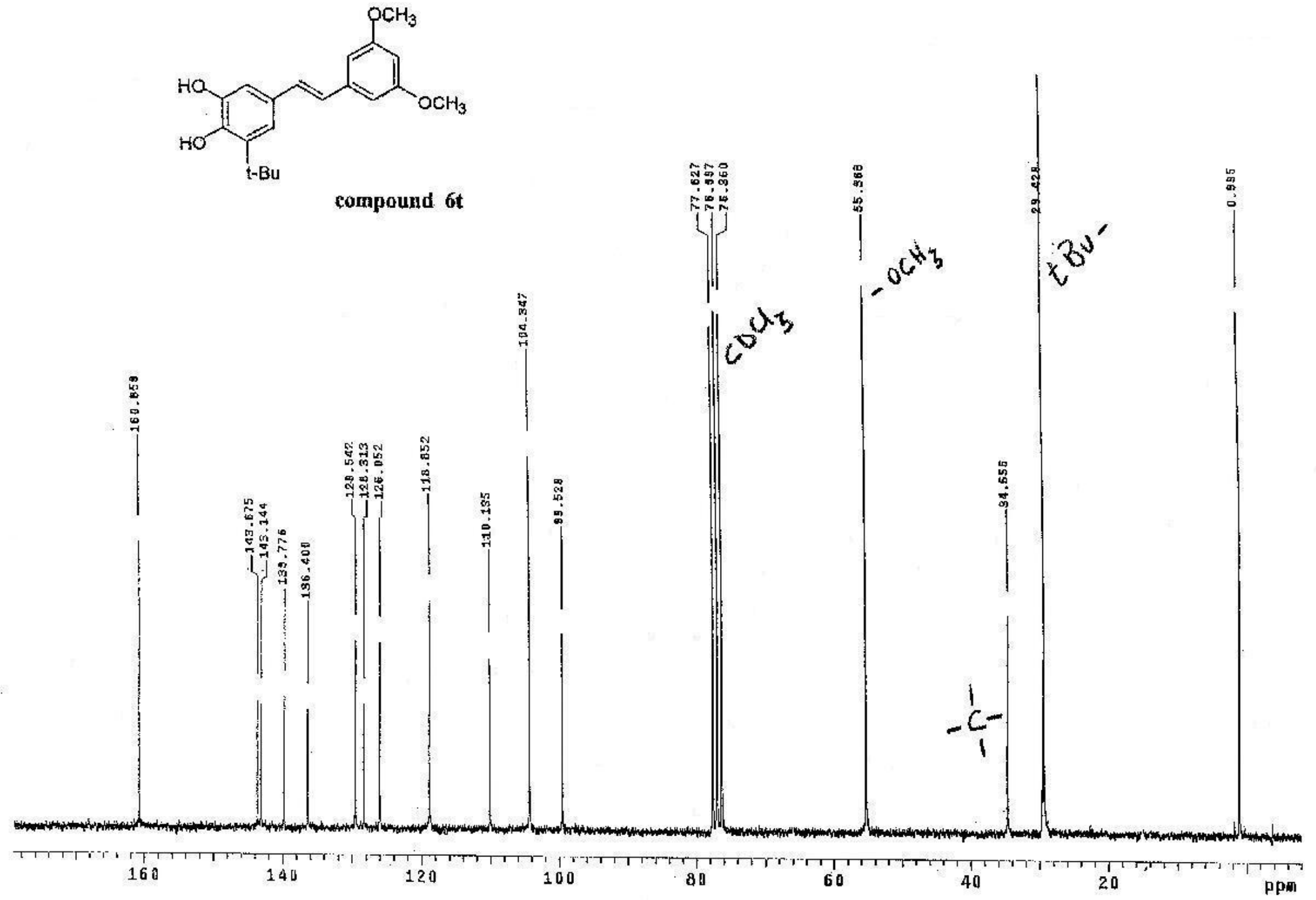


Table S1. Calculated $\Delta \mathrm{BDE}(\mathrm{O}-\mathrm{H})$ values with respect to phenol and energy difference between cis-trans isomers $(\Delta \mathrm{E} 1$ in parent phenols, $\Delta \mathrm{E} 2$ in radicals) of various stilbene derivatives.

\section{4-hydroxystilbene (12):}

\begin{tabular}{|l|l|l|}
\hline \multirow{2}{*}{ Molecule } & \multicolumn{2}{|c|}{$\Delta \mathrm{BDE}(\mathrm{O}-\mathrm{H})(\mathrm{kcal} / \mathrm{mol})$} \\
\cline { 2 - 3 } & uncorrected & $+\mathrm{ZPVE}$ \\
\hline trans-4-hydroxystilbene & -5.29 & -5.06 \\
\hline cis-4-hydroxystilbene & -4.03 & -3.92 \\
\hline$\Delta$ BDEcis - $\Delta$ BDEtrans & 1.26 & 1.14 \\
\hline
\end{tabular}

\begin{tabular}{|l|l|l|l|}
\hline \multicolumn{2}{|c|}{$\Delta \mathrm{E} 1(\mathrm{kcal} / \mathrm{mol})$} & \multicolumn{2}{c|}{$\Delta \mathrm{E} 2(\mathrm{kcal} / \mathrm{mol})$} \\
\hline uncorrected & + ZPVE & uncorrected & $+\mathrm{ZPVE}$ \\
\hline 5.02 & 5.30 & 6.28 & 6.45 \\
\hline
\end{tabular}

\section{Resveratrol (1)}

\begin{tabular}{|l|l|l|}
\hline \multirow{2}{*}{ Molecule } & \multicolumn{2}{|c|}{$\Delta \mathrm{BDE}(\mathrm{O}-\mathrm{H})(\mathrm{kcal} / \mathrm{mol})$} \\
\cline { 2 - 3 } & uncorrected & $+\mathrm{ZPVE}$ \\
\hline trans-resveratrol & -5.16 & -4.97 \\
\hline cis-resveratrol & -4.00 & -3.84 \\
\hline$\Delta$ BDEcis - $\Delta \mathrm{BDEtrans}$ & 1.16 & 1.13 \\
\hline
\end{tabular}

\begin{tabular}{|l|l|l|l|}
\hline \multicolumn{2}{|c|}{$\Delta \mathrm{E} 1(\mathrm{kcal} / \mathrm{mol})$} & \multicolumn{2}{c|}{$\Delta \mathrm{E} 2(\mathrm{kcal} / \mathrm{mol})$} \\
\hline uncorrected & + ZPVE & uncorrected & $+\mathrm{ZPVE}$ \\
\hline 5.34 & 5.52 & 6.50 & 6.66 \\
\hline
\end{tabular}


4'-hydroxy-3,5-dimethoxystilbene (2)

\begin{tabular}{|c|l|l|}
\hline \multirow{2}{*}{ Molecule } & \multicolumn{2}{|c|}{$\Delta \mathrm{BDE}(\mathrm{O}-\mathrm{H})(\mathrm{kcal} / \mathrm{mol})$} \\
\cline { 2 - 3 } & uncorrected & $+\mathrm{ZPVE}$ \\
\hline $\mathbf{2 t}$ & -5.42 & -5.22 \\
\hline $\mathbf{2 c}$ & -4.26 & -4.10 \\
\hline$\Delta$ BDEcis - $\Delta \mathrm{BDE}$ trans & 1.16 & 1.12 \\
\hline
\end{tabular}

\begin{tabular}{|l|l|l|l|}
\hline \multicolumn{2}{|c|}{$\Delta \mathrm{E} 1(\mathrm{kcal} / \mathrm{mol})$} & \multicolumn{2}{c|}{$\Delta \mathrm{E} 2(\mathrm{kcal} / \mathrm{mol})$} \\
\hline uncorrected & + ZPVE & uncorrected & $+\mathrm{ZPVE}$ \\
\hline 5.26 & 5.46 & 6.43 & 6.58 \\
\hline
\end{tabular}

Figure S1. Relevant distances ( $\AA$, bold) and spin density computed for $\mathbf{2 t}$ and $\mathbf{2 c}$ at the B3LYP/6-31G* level.<smiles>COc1cc(/C=C/[Al]=[V])cc(OC)c1</smiles><smiles>COc1cc(C=C(C)c2ccc(O)cc2)cc(OC)c1</smiles><smiles>COc1cc(/C=C/c2ccc(O)cc2)c([131I])c(OC)c1</smiles><smiles>COc1cc(C=Cc2ccc(O)cc2)cc(OC)c1</smiles> 


\section{Geometries and total energies from B3LYP/6-31G* calculations}

trans-4'-hydroxy-3,5-dimethoxystilbene

$\mathrm{E}(\mathrm{RB}+\mathrm{HF}-\mathrm{LYP})=-844.970916$ A.U.

Standard orientation:

\begin{tabular}{|c|c|c|c|c|c|}
\hline \multirow{2}{*}{$\begin{array}{l}\text { Center } \\
\text { Number }\end{array}$} & Atomic & \multicolumn{2}{|c|}{ Atomic } & \multicolumn{2}{|c|}{ Coordinates (Angstrom } \\
\hline & Numbe & & Type & $\begin{array}{ll}\mathrm{X} & \mathrm{Y}\end{array}$ & $\mathrm{Z}$ \\
\hline 1 & 6 & 0 & 5.666661 & -0.132307 & 0.000238 \\
\hline 2 & 8 & 0 & 7.015421 & -0.354624 & -0.000056 \\
\hline 3 & 1 & 0 & 7.474851 & 0.499541 & 0.006622 \\
\hline 4 & 6 & 0 & 5.107306 & 1.148226 & 0.006567 \\
\hline 5 & 6 & 0 & 4.826535 & -1.254169 & -0.006465 \\
\hline 6 & 6 & 0 & 3.722954 & 1.298200 & 0.006376 \\
\hline 7 & 6 & 0 & 2.856476 & 0.190965 & -0.000049 \\
\hline 8 & 6 & 0 & 3.449501 & -1.089455 & -0.006672 \\
\hline 9 & 1 & 0 & 2.821382 & -1.975188 & -0.012319 \\
\hline 10 & 1 & 0 & 5.273965 & -2.243210 & -0.011597 \\
\hline 11 & 1 & 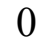 & 5.751855 & 2.025759 & 0.011564 \\
\hline 12 & 1 & 0 & 3.301042 & 2.300499 & 0.011408 \\
\hline 13 & 6 & 0 & 1.410940 & 0.421000 & 0.000361 \\
\hline 14 & 6 & 0 & 0.433517 & -0.508293 & -0.000911 \\
\hline 15 & 1 & 0 & 1.127874 & 1.472784 & 0.002347 \\
\hline 16 & 6 & 0 & -1.013450 & -0.264598 & -0.000727 \\
\hline 17 & 1 & 0 & 0.707881 & -1.562041 & -0.001833 \\
\hline 18 & 6 & 0 & -1.871595 & -1.375231 & 0.000890 \\
\hline
\end{tabular}




$\begin{array}{rrrrrr}19 & 6 & 0 & -3.257743 & -1.204617 & 0.001184 \\ 20 & 6 & 0 & -3.820064 & 0.078084 & 0.000123 \\ 21 & 6 & 0 & -2.958661 & 1.186841 & -0.001650 \\ 22 & 6 & 0 & -1.574761 & 1.023389 & -0.002099 \\ 23 & 1 & 0 & -1.475117 & -2.385673 & 0.001876 \\ 24 & 8 & 0 & -3.993143 & -2.357635 & 0.002722 \\ 25 & 1 & 0 & -4.892402 & 0.212656 & -0.000543 \\ 26 & 8 & 0 & -3.397872 & 2.482713 & -0.002963 \\ 27 & 1 & 0 & -0.959360 & 1.915700 & -0.003657 \\ 28 & 6 & 0 & -5.406452 & -2.260667 & 0.003910 \\ 29 & 1 & 0 & -5.777126 & -3.287558 & 0.005813 \\ 30 & 1 & 0 & -5.777107 & -1.741783 & 0.898358 \\ 31 & 1 & 0 & -5.778856 & -1.744525 & -0.891406 \\ 32 & 6 & 0 & -4.793015 & 2.727182 & -0.002305 \\ 33 & 1 & 0 & -4.906325 & 3.813083 & -0.003508 \\ 34 & 1 & 0 & -5.278868 & 2.313742 & -0.896622 \\ 35 & 1 & 0 & -5.277833 & 2.315293 & 0.893257 \\ -----------------------------------------------------------------\end{array}$

phenoxyl radical from trans-4'-hydroxy-3,5-dimethoxystilbene $\mathrm{E}(\mathrm{UB}+\mathrm{HF}-\mathrm{LYP})=-844.342331$ A.U.

Standard orientation:

\begin{tabular}{|c|c|c|c|c|}
\hline Center & Atomic & Atomic & Coordinate & s (Angstroms) \\
\hline Number & Number & Type & $\begin{array}{ll}X & Y\end{array}$ & $\mathrm{Z}$ \\
\hline 1 & 0 & -5.755862 & -0.145247 & -0.000107 \\
\hline
\end{tabular}




$\begin{array}{rrrrrr}2 & 8 & 0 & -6.993808 & -0.297012 & -0.000100 \\ 3 & 6 & 0 & -5.137401 & 1.176287 & -0.004422 \\ 4 & 6 & 0 & -4.837642 & -1.282353 & 0.004270 \\ 5 & 6 & 0 & -3.778061 & 1.322796 & -0.004381 \\ 6 & 6 & 0 & -2.895648 & 0.196717 & -0.000277 \\ 7 & 6 & 0 & -3.483386 & -1.110661 & 0.004164 \\ 8 & 1 & 0 & -2.836102 & -1.982858 & 0.007719 \\ 9 & 1 & 0 & -5.286156 & -2.271419 & 0.007710 \\ 10 & 1 & 0 & -5.805648 & 2.032172 & -0.007630 \\ 11 & 1 & 0 & -3.338108 & 2.318000 & -0.007682 \\ 12 & 6 & 0 & -1.480812 & 0.432180 & -0.000726 \\ 13 & 6 & 0 & -0.491507 & -0.509718 & 0.001290 \\ 14 & 1 & 0 & -1.192062 & 1.481610 & -0.003331 \\ 15 & 6 & 0 & 0.945394 & -0.265938 & 0.000832 \\ 16 & 1 & 0 & -0.770767 & -1.561107 & 0.003016 \\ 17 & 6 & 0 & 1.803230 & -1.379930 & -0.000020 \\ 18 & 6 & 0 & 3.188641 & -1.208130 & -0.000700 \\ 19 & 6 & 0 & 3.746964 & 0.077393 & -0.000648 \\ 20 & 6 & 0 & 2.887850 & 1.189037 & 0.000541 \\ 21 & 6 & 0 & 1.504107 & 1.025478 & 0.001205 \\ 22 & 1 & 0 & 1.405914 & -2.389781 & -0.000161 \\ 23 & 8 & 0 & 3.925025 & -2.356784 & -0.001566 \\ 24 & 1 & 0 & 4.819307 & 0.212241 & -0.000297 \\ 25 & 8 & 0 & 3.326813 & 2.481750 & 0.000553 \\ 26 & 1 & 0 & 0.888333 & 1.917140 & 0.002245 \\ 27 & 6 & 0 & 5.339981 & -2.258918 & -0.003082 \\ 28 & 1 & 0 & 5.709748 & -3.285766 & -0.004289 \\ 29 & 1 & 0 & 5.708755 & -1.741103 & -0.898519 \\ 30 & 1 & 0 & 5.710815 & -1.742531 & 0.892338 \\ 31 & 6 & 0 & 4.723114 & 2.730227 & 0.003096 \\ 32 & 1 & 0 & 4.832156 & 3.816203 & 0.004842 \\ 33 & 1 & 0 & 5.206504 & 2.318008 & 0.898869\end{array}$


cis-4'-hydroxy-3,5-dimethoxystilbene

$\mathrm{E}(\mathrm{RB}+\mathrm{HF}-\mathrm{LYP})=-844.962531$ A.U.

Standard orientation:

\begin{tabular}{|c|c|c|c|c|}
\hline \multirow{2}{*}{$\begin{array}{l}\text { Center } \\
\text { Number }\end{array}$} & Atomic & Atomic & \multicolumn{2}{|c|}{ Coordinates (Angstroms } \\
\hline & Numbe & r Type & $\begin{array}{ll}X & Y\end{array}$ & $\mathrm{Z}$ \\
\hline 1 & 6 & 4.317714 & 0.754766 & 0.593355 \\
\hline 2 & 8 & 5.197065 & 1.713494 & 1.014750 \\
\hline 3 & 1 & 6.038133 & 1.595038 & 0.546517 \\
\hline 4 & 6 & 4.646254 & -0.205653 & -0.367662 \\
\hline 5 & 6 & 3.042199 & 0.747367 & 1.171339 \\
\hline 6 & 6 & 3.700274 & -1.154608 & -0.747545 \\
\hline 7 & 6 & 2.403819 & -1.165913 & -0.204647 \\
\hline 8 & 6 & 2.106364 & -0.199544 & 0.777202 \\
\hline 9 & 1 & 1.128200 & -0.199326 & 1.245005 \\
\hline 10 & 1 & 2.806864 & 1.485179 & 1.932010 \\
\hline 11 & 1 & 5.639942 & -0.215535 & -0.812830 \\
\hline 12 & 1 & 3.971298 & -1.902068 & -1.489617 \\
\hline 13 & 6 & 1.470286 & -2.223468 & -0.625364 \\
\hline 14 & 6 & 0.121517 & -2.271863 & -0.664925 \\
\hline 15 & 1 & 1.973316 & -3.137375 & -0.943554 \\
\hline
\end{tabular}




$\begin{array}{rrrrrr}16 & 6 & 0 & -0.900419 & -1.233312 & -0.426120 \\ 17 & 1 & 0 & -0.303117 & -3.239938 & -0.930294 \\ 18 & 6 & 0 & -2.135393 & -1.631105 & 0.107776 \\ 19 & 6 & 0 & -3.152111 & -0.696202 & 0.313789 \\ 20 & 6 & 0 & -2.965507 & 0.650233 & -0.026546 \\ 21 & 6 & 0 & -1.737050 & 1.037722 & -0.582426 \\ 22 & 6 & 0 & -0.713435 & 0.110055 & -0.783710 \\ 23 & 1 & 0 & -2.319431 & -2.666083 & 0.378042 \\ 24 & 8 & 0 & -4.307515 & -1.190299 & 0.853656 \\ 25 & 1 & 0 & -3.754157 & 1.373055 & 0.127334 \\ 26 & 8 & 0 & -1.453017 & 2.318656 & -0.968855 \\ 27 & 1 & 0 & 0.212872 & 0.449295 & -1.231242 \\ 28 & 6 & 0 & -5.384310 & -0.298785 & 1.082963 \\ 29 & 1 & 0 & -6.187844 & -0.902984 & 1.508694 \\ 30 & 1 & 0 & -5.112403 & 0.492261 & 1.795075 \\ 31 & 1 & 0 & -5.735790 & 0.162700 & 0.150092 \\ 32 & 6 & 0 & -2.448032 & 3.313700 & -0.808482 \\ 33 & 1 & 0 & -2.007525 & 4.240236 & -1.181920 \\ 34 & 1 & 0 & -3.351219 & 3.083815 & -1.390187 \\ 35 & 1 & 0 & -2.724425 & 3.446575 & 0.246491 \\ --------------------------------------------------------------\end{array}$

phenoxyl radical from cis-4'-hydroxy-3,5-dimethoxystilbene

$\mathrm{E}(\mathrm{UB}+\mathrm{HF}-\mathrm{LYP})=-844.332091$ A.U.

Standard orientation: 


\begin{tabular}{|c|c|c|c|c|c|}
\hline \multirow{2}{*}{$\begin{array}{l}\text { Center } \\
\text { Number }\end{array}$} & \multirow{2}{*}{$\begin{array}{l}\text { Atomic } \\
\text { Number }\end{array}$} & \multirow{2}{*}{\multicolumn{2}{|c|}{$\begin{array}{l}\text { Atomic } \\
\text { Type }\end{array}$}} & \multicolumn{2}{|c|}{ Coordinates (Angstron } \\
\hline & & & & X $\quad Y$ & $\mathrm{Z}$ \\
\hline 1 & 6 & 0 & 4.437832 & 0.845149 & 0.641495 \\
\hline 2 & 8 & 0 & 5.279857 & 1.692455 & 1.005490 \\
\hline 3 & 6 & 0 & 4.778991 & -0.241603 & -0.269030 \\
\hline 4 & 6 & 0 & 3.057765 & 0.874366 & 1.114984 \\
\hline 5 & 6 & 0 & 3.841563 & -1.159055 & -0.658690 \\
\hline 6 & 6 & 0 & 2.481335 & -1.082549 & -0.223114 \\
\hline 7 & 6 & 0 & 2.134004 & -0.043170 & 0.698054 \\
\hline 8 & 1 & 0 & 1.121477 & -0.002166 & 1.082471 \\
\hline 9 & 1 & 0 & 2.80 & 1.655415 & 1.824507 \\
\hline 10 & 1 & 0 & 306446 & -0.289179 & -0.617339 \\
\hline 11 & 1 & 0 & 119400 & -1.965370 & -1.334401 \\
\hline 12 & 6 & 0 & 1.571323 & -2.109287 & -0.674597 \\
\hline 13 & 6 & 0 & 0.208 & -2.198112 & -0.681279 \\
\hline 14 & 1 & 0 & 2.0802 & -2.993794 & -1.058474 \\
\hline 15 & 6 & 0 & -0.840817 & -1.201370 & -0.432934 \\
\hline 16 & 1 & & -0.184006 & -3.179328 & -0.947171 \\
\hline 17 & 6 & & -2.063855 & -1.649283 & 0.093103 \\
\hline 18 & 6 & & -3.1087 & -0.749707 & 0.312788 \\
\hline 19 & 6 & 0 & -2.9642 & 0.606141 & -0.014860 \\
\hline 20 & 6 & 0 & -1.751749 & 1.042812 & -0.569800 \\
\hline 21 & 6 & 0 & -0.697061 & 0.151773 & -0.779173 \\
\hline 22 & 1 & 0 & -2.212862 & -2.692645 & 0.351571 \\
\hline 23 & 8 & 0 & -4.244275 & -1.284378 & 0.848563 \\
\hline 24 & 1 & 0 & -3.776603 & 1.300702 & 0.146201 \\
\hline 25 & 8 & 0 & -1.512493 & 2.332249 & -0.946191 \\
\hline 26 & 1 & & 0.2129 & 0.524428 & -1.233438 \\
\hline 27 & 6 & & -5.349526 & -0.430061 & 1.094270 \\
\hline 28 & 1 & 0 & -6.129194 & -1.065634 & 1.517809 \\
\hline
\end{tabular}




$\begin{array}{rrrrrr}29 & 1 & 0 & -5.097787 & 0.360475 & 1.813720 \\ 30 & 1 & 0 & -5.720954 & 0.028412 & 0.168092 \\ 31 & 6 & 0 & -2.539527 & 3.295367 & -0.776671 \\ 32 & 1 & 0 & -2.128311 & 4.237057 & -1.144369 \\ 33 & 1 & 0 & -3.434754 & 3.039672 & -1.359208 \\ 34 & 1 & 0 & -2.816193 & 3.410887 & 0.279864 \\ 34 & 1 & 0 & -2.816193 & 3.410887 & 0.279864 \\ 34 & 1 & 0 & -2.816193 & 3.410887 & 0.279864\end{array}$

trans-resveratrol

$\mathrm{E}(\mathrm{RB}+\mathrm{HF}-\mathrm{LYP})=-766.358219$ A.U.

Standard orientation:

\begin{tabular}{|c|c|c|c|c|}
\hline \multirow{2}{*}{$\begin{array}{l}\text { Center } \\
\text { Number }\end{array}$} & \multirow{2}{*}{$\begin{array}{l}\text { Atomic } \\
\text { Numbe }\end{array}$} & \multirow{2}{*}{$\begin{array}{r}\text { Atomic } \\
\text { Type }\end{array}$} & \multicolumn{2}{|c|}{ Coordinates (Angstrom } \\
\hline & & & X $\quad Y$ & Z \\
\hline 1 & 6 & -4.963310 & -0.135404 & -0.000082 \\
\hline 2 & 8 & -6.309850 & -0.368855 & 0.000080 \\
\hline 3 & 1 & -6.776787 & 0.481288 & -0.003258 \\
\hline 4 & 6 & -4.414245 & 1.149715 & -0.005019 \\
\hline 5 & 6 & -4.114318 & -1.250753 & 0.005028 \\
\hline 6 & 6 & -3.031189 & 1.310640 & -0.004894 \\
\hline 7 & 6 & -2.156200 & 0.210065 & -0.000046 \\
\hline 8 & 6 & -2.738765 & -1.075157 & 0.005054 \\
\hline
\end{tabular}




$\begin{array}{ccrrrr}9 & 1 & 0 & -2.103709 & -1.955919 & 0.009385 \\ 10 & 1 & 0 & -4.554001 & -2.243243 & 0.009064 \\ 11 & 1 & 0 & -5.065754 & 2.022051 & -0.008935 \\ 12 & 1 & 0 & -2.617188 & 2.316227 & -0.008788 \\ 13 & 6 & 0 & -0.712774 & 0.451123 & -0.000392 \\ 14 & 6 & 0 & 0.271279 & -0.471380 & 0.000629 \\ 15 & 1 & 0 & -0.437761 & 1.505007 & -0.001985 \\ 16 & 6 & 0 & 1.715933 & -0.217428 & 0.000466 \\ 17 & 1 & 0 & 0.004128 & -1.526830 & 0.001299 \\ 18 & 6 & 0 & 2.579772 & -1.325622 & -0.001539 \\ 19 & 6 & 0 & 3.962493 & -1.142896 & -0.001954 \\ 20 & 6 & 0 & 4.510142 & 0.142731 & -0.000326 \\ 21 & 6 & 0 & 3.647382 & 1.246636 & 0.001770 \\ 22 & 6 & 0 & 2.266066 & 1.077180 & 0.002239 \\ 23 & 1 & 0 & 2.187207 & -2.337373 & -0.002921 \\ 24 & 8 & 0 & 4.743119 & -2.266921 & -0.003998 \\ 25 & 1 & 0 & 5.590562 & 0.284795 & -0.000501 \\ 26 & 8 & 0 & 4.120641 & 2.531521 & 0.003696 \\ 27 & 1 & 0 & 1.642324 & 1.963468 & 0.004168 \\ 28 & 1 & 0 & 5.676303 & -2.004163 & -0.004179 \\ 29 & 1 & 0 & 5.089799 & 2.512031 & 0.002827 \\ --------------------------------------------------------------\end{array}$

phenoxyl radical from trans-resveratrol

$\mathrm{E}(\mathrm{UB}+\mathrm{HF}-\mathrm{LYP})=-765.729206$ A.U.

Standard orientation: 


\begin{tabular}{|c|c|c|c|c|}
\hline \multirow{2}{*}{$\begin{array}{l}\text { Center } \\
\text { Number }\end{array}$} & Atomic & Atomic & \multicolumn{2}{|c|}{ Coordinates (Angstron } \\
\hline & Numbe & r Type & X $\quad Y$ & Z \\
\hline 1 & 6 & 5.055768 & -0.149896 & -0.000102 \\
\hline 2 & 8 & 6.292182 & -0.312146 & -0.000401 \\
\hline 3 & 6 & 4.448410 & 1.176902 & 0.001410 \\
\hline 4 & 6 & 4.128298 & -1.279672 & -0.001376 \\
\hline 5 & 6 & 3.090321 & 1.334604 & 0.001583 \\
\hline 6 & 6 & 2.198954 & 0.215605 & 0.000340 \\
\hline 7 & 6 & 2.775616 & -1.096805 & -0.001149 \\
\hline 8 & 1 & 2.121168 & -1.963613 & -0.002192 \\
\hline 9 & 1 & 4.568762 & -2.272324 & -0.002554 \\
\hline 10 & 1 & 5.123672 & 2.027235 & 0.002359 \\
\hline 11 & 1 & 2.658589 & 2.333383 & 0.002701 \\
\hline 12 & 6 & 0.786032 & 0.462393 & 0.000542 \\
\hline 13 & 6 & -0.210134 & -0.472278 & -0.000211 \\
\hline 14 & 1 & 0.505800 & 1.514101 & 0.001417 \\
\hline 15 & 6 & -1.645011 & -0.218022 & -0.000162 \\
\hline 16 & 1 & 0.061511 & -1.525517 & -0.000804 \\
\hline 17 & 6 & -2.508965 & -1.329080 & 0.000336 \\
\hline 18 & 6 & -3.890983 & -1.145029 & 0.000407 \\
\hline 19 & 6 & -4.434426 & 0.143473 & -0.000095 \\
\hline 20 & 6 & -3.573408 & 1.249770 & -0.000610 \\
\hline 21 & 6 & -2.192306 & 1.079886 & -0.000695 \\
\hline 22 & 1 & -2.115959 & -2.340434 & 0.000697 \\
\hline 23 & 8 & -4.671186 & -2.265979 & 0.001273 \\
\hline 24 & 1 & -5.514517 & 0.286361 & 0.000115 \\
\hline 25 & 8 & -4.044763 & 2.532219 & -0.001087 \\
\hline 26 & 1 & -1.568055 & 1.965456 & -0.001354 \\
\hline 27 & 1 & -5.014161 & 2.518896 & -0.001133 \\
\hline 28 & 1 & -5.605671 & -2.007396 & 0.001162 \\
\hline
\end{tabular}


cis-resveratrol

$\mathrm{E}(\mathrm{RB}+\mathrm{HF}-\mathrm{LYP})=-766.349705$ A.U.

Standard orientation:

\begin{tabular}{|c|c|c|c|c|c|}
\hline \multirow{2}{*}{$\begin{array}{l}\text { Center } \\
\text { Number }\end{array}$} & \multirow{2}{*}{$\begin{array}{l}\text { Atomic } \\
\text { Number }\end{array}$} & \multirow{2}{*}{\multicolumn{2}{|c|}{$\begin{array}{l}\text { Atomic } \\
\text { Type }\end{array}$}} & \multicolumn{2}{|c|}{ Coordinates (Angstroms) } \\
\hline & & & & $\mathrm{X} \quad \mathrm{Y}$ & $\mathrm{Z}$ \\
\hline 1 & 6 & 0 & 3.858501 & 0.707747 & 0.406352 \\
\hline 2 & 8 & 0 & 4.827177 & 1.627930 & 0.696174 \\
\hline 3 & 1 & 0 & 5.633228 & 1.400393 & 0.207069 \\
\hline 4 & 6 & 0 & 4.071790 & -0.371999 & -0.455526 \\
\hline 5 & 6 & 0 & 2.610656 & 0.866447 & 1.022008 \\
\hline 6 & 6 & 0 & 3.040392 & -1.275728 & -0.698444 \\
\hline 7 & 6 & 0 & 1.770556 & -1.123240 & -0.115675 \\
\hline 8 & 6 & 0 & 1.588455 & -0.036645 & 0.763103 \\
\hline 9 & 1 & 0 & 0.632948 & 0.092779 & 1.259144 \\
\hline 10 & 1 & 0 & 2.465095 & 1.697586 & 1.704919 \\
\hline 11 & 1 & 0 & 5.042812 & -0.510413 & -0.928296 \\
\hline 12 & 1 & 0 & 3.221877 & -2.117698 & -1.362515 \\
\hline 13 & 6 & 0 & 0.741272 & -2.140884 & -0.383097 \\
\hline 14 & 6 & 0 & -0.607418 & -2.078699 & -0.377409 \\
\hline 15 & 1 & 0 & 1.156653 & -3.125126 & -0.602617 \\
\hline 16 & 6 & 0 & -1.528418 & -0.932908 & -0.240769 \\
\hline 17 & 1 & 0 & -1.120686 & -3.030923 & -0.510117 \\
\hline 18 & 6 & 0 & -2.767520 & -1.151549 & 0.383140 \\
\hline 19 & 6 & 0 & -3.691037 & -0.112012 & 0.493020 \\
\hline 20 & 6 & 0 & -3.404657 & 1.152161 & -0.031047 \\
\hline
\end{tabular}




$\begin{array}{rrrrrr}21 & 6 & 0 & -2.177591 & 1.359894 & -0.672190 \\ 22 & 6 & 0 & -1.242789 & 0.331233 & -0.781120 \\ 23 & 1 & 0 & -3.021264 & -2.123420 & 0.793982 \\ 24 & 8 & 0 & -4.873523 & -0.387096 & 1.124807 \\ 25 & 1 & 0 & -4.129951 & 1.961663 & 0.049466 \\ 26 & 8 & 0 & -1.845814 & 2.568057 & -1.223189 \\ 27 & 1 & 0 & -0.311453 & 0.523204 & -1.299709 \\ 28 & 1 & 0 & -5.423424 & 0.411372 & 1.130000 \\ 29 & 1 & 0 & -2.581700 & 3.186560 & -1.097743\end{array}$

phenoxyl radical from cis-resveratrol

$\mathrm{E}(\mathrm{UB}+\mathrm{HF}-\mathrm{LYP})=-765.718845$

Standard orientation:

\begin{tabular}{|c|c|c|c|c|}
\hline \multirow{2}{*}{$\begin{array}{l}\text { Center } \\
\text { Number }\end{array}$} & \multirow{2}{*}{$\begin{array}{l}\text { Atomic } \\
\text { Numbe }\end{array}$} & \multirow{2}{*}{$\begin{array}{r}\text { Atomic } \\
\text { Type }\end{array}$} & \multicolumn{2}{|c|}{ Coordinates (Angstroms } \\
\hline & & & $\begin{array}{ll}X & Y\end{array}$ & $\mathrm{Z}$ \\
\hline 1 & 6 & 3.987238 & 0.797835 & 0.436123 \\
\hline 2 & 8 & 4.909461 & 1.603485 & 0.680054 \\
\hline 3 & 6 & 4.204813 & -0.407338 & -0.355512 \\
\hline 4 & 6 & 2.632437 & 0.997531 & 0.940564 \\
\hline 5 & 6 & 3.183203 & -1.281786 & -0.608219 \\
\hline 6 & 6 & 1.851862 & -1.044057 & -0.142767 \\
\hline 7 & 6 & 1.622904 & 0.119526 & 0.659002 \\
\hline
\end{tabular}




$\begin{array}{cccccc}8 & 1 & 0 & 0.632383 & 0.288747 & 1.065181 \\ 9 & 1 & 0 & 2.466208 & 1.872797 & 1.561606 \\ 10 & 1 & 0 & 5.210890 & -0.579066 & -0.726307 \\ 11 & 1 & 0 & 3.369373 & -2.179647 & -1.193968 \\ 12 & 6 & 0 & 0.848405 & -2.040427 & -0.437821 \\ 13 & 6 & 0 & -0.516222 & -2.019507 & -0.391997 \\ 14 & 1 & 0 & 1.272385 & -3.003333 & -0.723699 \\ 15 & 6 & 0 & -1.475181 & -0.917730 & -0.242032 \\ 16 & 1 & 0 & -0.992904 & -2.990858 & -0.520791 \\ 17 & 6 & 0 & -2.707088 & -1.197952 & 0.375042 \\ 18 & 6 & 0 & -3.669124 & -0.196522 & 0.501806 \\ 19 & 6 & 0 & -3.431383 & 1.085234 & -0.006395 \\ 20 & 6 & 0 & -2.215634 & 1.352903 & -0.646980 \\ 21 & 6 & 0 & -1.239308 & 0.364167 & -0.767652 \\ 22 & 1 & 0 & -2.921597 & -2.184829 & 0.771692 \\ 23 & 8 & 0 & -4.836609 & -0.526063 & 1.129690 \\ 24 & 1 & 0 & -4.189098 & 1.863030 & 0.083882 \\ 25 & 8 & 0 & -1.932791 & 2.575930 & -1.184972 \\ 26 & 1 & 0 & -0.321561 & 0.597782 & -1.293344 \\ 27 & 1 & 0 & -2.688255 & 3.169002 & -1.052175 \\ 28 & 1 & 0 & -5.419831 & 0.248292 & 1.150769 \\ ------------------------------------------------------------------\end{array}$


trans-4-hydroxystilbene

$\mathrm{E}(\mathrm{RB}+\mathrm{HF}-\mathrm{LYP})=-615.926476$ A.U.

Standard orientation:

\begin{tabular}{|c|c|c|c|c|c|}
\hline \multirow{2}{*}{$\begin{array}{l}\text { Center } \\
\text { Number }\end{array}$} & Atomic & \multicolumn{2}{|c|}{ Atomic } & \multicolumn{2}{|c|}{ Coordinates (Angstrom } \\
\hline & Numbe & & Type & $\begin{array}{ll}X & Y\end{array}$ & $\mathrm{Z}$ \\
\hline 1 & 6 & 0 & -4.266826 & -0.116130 & -0.000116 \\
\hline 2 & 8 & 0 & -5.613007 & -0.351686 & 0.000103 \\
\hline 3 & 1 & 0 & -6.081415 & 0.497631 & -0.003675 \\
\hline 4 & 6 & 0 & -3.719802 & 1.169803 & -0.004664 \\
\hline 5 & 6 & 0 & -3.415810 & -1.229846 & 0.004724 \\
\hline 6 & 6 & 0 & -2.337025 & 1.333092 & -0.004467 \\
\hline 7 & 6 & 0 & -1.459792 & 0.234244 & 0.000118 \\
\hline 8 & 6 & 0 & -2.040496 & -1.051848 & 0.004900 \\
\hline 9 & 1 & 0 & -1.404120 & -1.931713 & 0.009137 \\
\hline 10 & 1 & 0 & -3.853643 & -2.223154 & 0.008501 \\
\hline 11 & 1 & 0 & -4.372714 & 2.041083 & -0.008304 \\
\hline 12 & 1 & 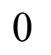 & -1.925054 & 2.339561 & -0.008069 \\
\hline 13 & 6 & 0 & -0.016686 & 0.477820 & -0.000079 \\
\hline 14 & 6 & 0 & 0.969734 & -0.442833 & -0.000019 \\
\hline 15 & 1 & 0 & 0.255012 & 1.532770 & -0.000702 \\
\hline 16 & 6 & 0 & 2.414093 & -0.192270 & 0.000146 \\
\hline 17 & 1 & 0 & 0.701147 & -1.498498 & -0.000681 \\
\hline 18 & 6 & 0 & 3.291518 & -1.293229 & -0.004575 \\
\hline
\end{tabular}




$\begin{array}{rrrrrr}19 & 6 & 0 & 4.674277 & -1.120655 & -0.004835 \\ 20 & 6 & 0 & 5.219408 & 0.163582 & -0.000311 \\ 21 & 6 & 0 & 4.364388 & 1.270231 & 0.004590 \\ 22 & 6 & 0 & 2.984185 & 1.096849 & 0.005091 \\ 23 & 1 & 0 & 2.875216 & -2.298238 & -0.008194 \\ 24 & 1 & 0 & 6.296942 & 0.303500 & -0.000838 \\ 25 & 1 & 0 & 2.342304 & 1.972923 & 0.009142 \\ 26 & 1 & 0 & 5.325591 & -1.990849 & -0.008544 \\ 27 & 1 & 0 & 4.777797 & 2.275610 & 0.008386\end{array}$

phenoxyl radical from trans-4-hydroxystilbene

$\mathrm{E}(\mathrm{UB}+\mathrm{HF}-\mathrm{LYP})=-615.297675$ A.U.

Standard orientation:

\begin{tabular}{llllrr} 
Center & \multicolumn{2}{c}{ Atomic } & Atomic & \multicolumn{2}{c}{ Coordinates (Angstrom } \\
Number & Number & Type & \multicolumn{1}{c}{ X } & Y & Z \\
\hline 1 & 6 & 0 & -4.363514 & -0.129587 & 0.000054 \\
2 & 8 & 0 & -5.599652 & -0.293219 & 0.000284 \\
3 & 6 & 0 & -3.757757 & 1.197985 & -0.001995 \\
4 & 6 & 0 & -3.434326 & -1.258016 & 0.001926 \\
5 & 6 & 0 & -2.400020 & 1.357490 & -0.002078
\end{tabular}




$\begin{array}{cccrrr}6 & 6 & 0 & -1.506687 & 0.239859 & -0.000269 \\ 7 & 6 & 0 & -2.081941 & -1.073278 & 0.001765 \\ 8 & 1 & 0 & -1.426509 & -1.939398 & 0.003354 \\ 9 & 1 & 0 & -3.873340 & -2.251314 & 0.003529 \\ 10 & 1 & 0 & -4.434150 & 2.047422 & -0.003400 \\ 11 & 1 & 0 & -1.969930 & 2.357032 & -0.003602 \\ 12 & 6 & 0 & -0.094720 & 0.488587 & -0.000470 \\ 13 & 6 & 0 & 0.904245 & -0.444481 & 0.000225 \\ 14 & 1 & 0 & 0.182391 & 1.541299 & -0.001494 \\ 15 & 6 & 0 & 2.337548 & -0.193219 & 0.000231 \\ 16 & 1 & 0 & 0.631152 & -1.497873 & 0.000490 \\ 17 & 6 & 0 & 3.216213 & -1.297213 & -0.001887 \\ 18 & 6 & 0 & 4.596824 & -1.122373 & -0.002113 \\ 19 & 6 & 0 & 5.138224 & 0.164726 & -0.000187 \\ 20 & 6 & 0 & 4.284055 & 1.273155 & 0.002083 \\ 21 & 6 & 0 & 2.905259 & 1.100041 & 0.002513 \\ 22 & 1 & 0 & 2.799704 & -2.301786 & -0.003466 \\ 23 & 1 & 0 & 6.215423 & 0.305670 & -0.000712 \\ 24 & 1 & 0 & 2.262751 & 1.975143 & 0.004349 \\ 25 & 1 & 0 & 5.250721 & -1.990088 & -0.003769 \\ 26 & 1 & 0 & 4.698595 & 2.277592 & 0.003676 \\ -------------------------------------------------------------------\end{array}$


cis-4-hydroxystilbene

$$
\mathrm{E}(\mathrm{RB}+\mathrm{HF}-\mathrm{LYP})=-615.918479 \text { A.U. }
$$

Standard orientation:

\begin{tabular}{cccccc} 
Center & \multicolumn{2}{c}{ Atomic } & \multicolumn{2}{c}{ Atomic } & \multicolumn{3}{c}{ Coordinates (Angstroms) } \\
Number & Number & Type & X & Y & $Z$ \\
\hline 1 & 6 & 0 & 3.381239 & -0.736917 & -0.140118 \\
2 & 8 & 0 & 4.408116 & -1.636233 & -0.215075 \\
3 & 1 & 0 & 5.184672 & -1.265358 & 0.232278 \\
4 & 6 & 0 & 3.501631 & 0.500476 & 0.499090 \\
5 & 6 & 0 & 2.168950 & -1.087133 & -0.747820 \\
6 & 6 & 0 & 2.414529 & 1.370500 & 0.530731 \\
7 & 6 & 0 & 1.178513 & 1.031496 & -0.046165 \\
8 & 6 & 0 & 1.090312 & -0.214119 & -0.699784 \\
9 & 1 & 0 & 0.164165 & -0.494262 & -1.189758 \\
10 & 1 & 0 & 2.095892 & -2.041210 & -1.260505 \\
11 & 1 & 0 & 4.444460 & 0.786424 & 0.962596 \\
12 & 1 & 0 & 2.524439 & 2.334793 & 1.021499 \\
13 & 6 & 0 & 0.084520 & 2.016191 & -0.011635 \\
14 & 6 & 0 & -1.257385 & 1.865133 & -0.032672 \\
15 & 1 & 0 & 0.434302 & 3.048760 & 0.015839 \\
16 & 6 & 0 & -2.100691 & 0.657327 & 0.045279 \\
17 & 1 & 0 & -1.830193 & 2.790019 & -0.104724 \\
18 & 6 & 0 & -3.341530 & 0.651772 & -0.617868 \\
19 & 6 & 0 & -4.197593 & -0.445505 & -0.540275 \\
20 & 6 & 0 & -3.840344 & -1.559187 & 0.221547 \\
& & & & &
\end{tabular}




$\begin{array}{rrrrrr}21 & 6 & 0 & -2.622483 & -1.560353 & 0.906877 \\ 22 & 6 & 0 & -1.763927 & -0.466570 & 0.822465 \\ 23 & 1 & 0 & -3.630341 & 1.520409 & -1.205476 \\ 24 & 1 & 0 & -4.508077 & -2.413829 & 0.289782 \\ 25 & 1 & 0 & -0.828681 & -0.472061 & 1.372694 \\ 26 & 1 & 0 & -5.146640 & -0.427818 & -1.069917 \\ 27 & 1 & 0 & -2.343377 & -2.414652 & 1.518377\end{array}$

phenoxyl radical from cis-4-hydroxystilbene

$E(U B+H F-L Y P)=-615.287671$ A.U.

Standard orientation:

\begin{tabular}{lllrrr} 
Center & \multicolumn{2}{c}{ Atomic } & \multicolumn{2}{c}{ Atomic } & \multicolumn{2}{c}{ Coordinates (Angstroms) } \\
Number & Number & Type & X & Y & Z \\
\hline 1 & 6 & 0 & 3.501344 & -0.834614 & -0.145105 \\
2 & 8 & 0 & 4.468778 & -1.622314 & -0.191276 \\
3 & 6 & 0 & 3.626864 & 0.511272 & 0.402150 \\
4 & 6 & 0 & 2.181837 & -1.200180 & -0.651023 \\
5 & 6 & 0 & 2.555814 & 1.361805 & 0.442623 \\
6 & 6 & 0 & 1.258838 & 0.966620 & -0.012860 \\
7 & 6 & 0 & 1.121503 & -0.339949 & -0.582398
\end{tabular}




$\begin{array}{cccccc}8 & 1 & 0 & 0.160902 & -0.635718 & -0.989030 \\ 9 & 1 & 0 & 2.085620 & -2.184957 & -1.098759 \\ 10 & 1 & 0 & 4.606165 & 0.805568 & 0.767576 \\ 11 & 1 & 0 & 2.673663 & 2.364729 & 0.847752 \\ 12 & 6 & 0 & 0.195808 & 1.941781 & 0.049109 \\ 13 & 6 & 0 & -1.164010 & 1.827534 & -0.025192 \\ 14 & 1 & 0 & 0.557002 & 2.965840 & 0.146549 \\ 15 & 6 & 0 & -2.052115 & 0.663316 & 0.037814 \\ 16 & 1 & 0 & -1.696813 & 2.774486 & -0.112304 \\ 17 & 6 & 0 & -3.298515 & 0.729186 & -0.617178 \\ 18 & 6 & 0 & -4.199734 & -0.330206 & -0.556837 \\ 19 & 6 & 0 & -3.888409 & -1.471859 & 0.185336 \\ 20 & 6 & 0 & -2.671555 & -1.540545 & 0.869534 \\ 21 & 6 & 0 & -1.763924 & -0.487308 & 0.799154 \\ 22 & 1 & 0 & -3.549633 & 1.620611 & -1.187184 \\ 23 & 1 & 0 & -4.594038 & -2.296068 & 0.241942 \\ 24 & 1 & 0 & -0.834090 & -0.539845 & 1.355800 \\ 25 & 1 & 0 & -2.432995 & -2.414687 & 1.469267 \\ 26 & 1 & 0 & -5.148480 & -0.262566 & -1.082169 \\ --------------------------------------------------------------\end{array}$

